\title{
Actividad física intensa: Dulce o amargo
}

\section{Sr. Editor:}

Según la OMS se considera actividad física cualquier movimiento corporal producido por los músculos esqueléticos que exija gasto de energía. Por otro lado, la actividad física intensa (aproximadamente $>6$ MET u equivalentes metabólicos) es aquella que requiere una gran cantidad de esfuerzo y provoca una respiración rápida y un aumento sustancial de la frecuencia cardíaca, por ejemplo, deportes y juegos competitivos, trabajo intenso con pala, excavación de zanjas o desplazamiento de cargas pesadas (> $20 \mathrm{Kg}$.). Tiene beneficios sobre el aparato cardiovascular y muscular, pero también sobre el metabolismo, el sistema endocrino y el sistema inmunológico, para mantener un buen peso y en general para la salud ${ }^{1}$.

En ocasiones pensamos que haciendo actividad física intensa vamos a llegar a nuestro objetivo rápidamente, pero no tenemos en cuenta las consecuencias que esto puede ocasionar, como infarto en pacientes con colesterol alto, hipertensión, diabetes, obesidad y fumadores. Sobre todo, si estas personas son sedentarias y de repente realizan una actividad intensa sin tener una condición física apropiada. También mantener una actividad laboral físicamente intensa durante años aumenta el riesgo de padecer una arritmia, especialmente la más frecuente de ellas, la fibrilación auricular, según un estudio del Hospital Clínico de Barcelona. Esta información no sólo debe ser conocida por el personal de salud, sino ser motivo de información en y para la familia, colegios y centros de trabajo, por lo que sugerimos que se incluya en la promoción y prevención en salud.

Así pues, ante estos efectos adversos, la OMS establece recomendaciones para la duración de la actividad física intensa y sobre cómo deberían ser los parámetros según las edades. Los niños y jóvenes de 5 a 17 años deberían invertir como mínimo 60 minutos diarios en actividad física de intensidad moderada a vigorosa y la actividad física diaria debería ser, en su mayor parte, aeróbica. Convendría incorporar, como mínimo tres veces por semana, actividades vigorosas que refuercen, en particular, los músculos y huesos. Los adultos de 18 a 64 años deberían dedicar como mínimo
150 minutos semanales a la práctica de actividad física aeróbica, de intensidad moderada, o bien 75 minutos de actividad física aeróbica vigorosa cada semana, o bien una combinación equivalente de actividades moderadas y vigorosas. Para obtener aún mayores beneficios para la salud, los adultos de este grupo de edades deberían aumentar hasta 300 minutos por semana la práctica de actividad física moderada aeróbica, o bien hasta 150 minutos semanales de actividad física intensa aeróbica, o una combinación equivalente de actividad moderada y vigorosa ${ }^{2}$.

Además, la Federación Española de Medicina del Deporte $^{3}$ afirma que "a pesar de que el deporte de alta intensidad tiene consecuencias sobre el organismo y especialmente sobre el aparato cardiovascular, si se realiza en unas condiciones de cuidado y de vigilancia adecuadas, se pueden minimizar los riesgos". Por todo lo mencionado, se concluye que es necesario recomendar a todos, deportistas o no, realizarse una continua evaluación de su salud y también de sus parámetros físicos fundamentales cuando se realice una actividad física intensa, y si es necesario teniendo en cuenta programas de actividades adaptadas a las preferencias y al estilo de vida de la persona. Debe tenerse en cuenta que "todo en exceso hace daño".

\section{BIBLIOGRAFÍA}

1. Organización mundial de salud, Recomendaciones mundiales sobre la actividad física para la salud, Noviembre 2010. Disponible en: http://www.who.int/dietphysicalactivity/ factsheet_recommendations/es/index.html.

2. Kelishadi R, Ardalan G, Asociación de la actividad física y los hábitos alimentarios en relación con el índice de masa corporal en una muestra nacional de niños y adolescentes iraníes: estudio CASPIAN, Boletín de la Organización Mundial de la Salud. 2007; 85:1-84.

3. Boraita A. Guias de practica clinica sobre actividad fisica del cardiopata, Rev Esp Cardiol. 2000; 53:684-726.

María Isabel Bravo Bartra

Estudiante de la Escuela de Tecnología Medica, Terapia Física y Rehabilitación.

Facultad de Medicina Alberto Hurtado, Universidad Peruana Cayetano Heredia, Lima, Perú 